\title{
Point of Care (POC) for Early Infant Diagnosis (EID) in Nigeria? Healthcare Workers Opinion
}

\author{
Busari Olusegun \\ ${ }^{1}$ Department of Public Health, Texila American University, Guyana
}

\begin{abstract}
Without access to life-saving drugs, including antiretroviral, about one-third of HIV exposed infants (HEI) will die by age 1 year and 50\% by age 2 years. A $75 \%$ reduction in disease progression and $76 \%$ reduction in HIV mortality in infants has been attributed to early diagnosis of HIV and early commencement on ART. Early Infant Diagnosis (EID) of HIV aids timely commencement on antiretroviral therapy $(A R T)$. Several challenges have been identified with the current process. Point of care (POC) technologies are recommended as a veritable means of addressing these challenges and improving EID uptake. With the aim of assessing the standard of care and the acceptability of POC for the provision of EID. A descriptive cross-sectional survey was conducted across eight healthcare facilities in Nigeria. The survey was conducted among 72 healthcare workers using self-administered questionnaire; with a recovery rate of $61(84.7 \%)$. Analysis of participants' responses indicate that $100 \%$ of the respondents believes there is a need for EID. Most respondent reported an average turnaround time (TAT) of 3-4 weeks (35.8\%) and >6 weeks (34.0\%). Most respondents identified distance to the PCR laboratories (45.7\%) and long TAT (34.8\%) as key issues affecting the conduct of EID. On the benefit of POC for EID; $90.2 \%$ of respondent believe it is beneficial; while $81.5 \%$ of respondent believe that with the introduction and use of POC for EID there will be an increase in EID uptake. The POC is a viable and acceptable alternative for EID to increase uptake.
\end{abstract}

Keywords: Early Infant Diagnosis, HIV/AIDS, Point of Care.

\section{Introduction}

Despite four (4) decades of the fight against the HIV/AIDS epidemic, the scourge, its impact, and its effects are still with us. Infants and children are one of the population groups that are highly affected by the HIV epidemic due to the aggressive course of the infection in them. About one-third of infants will die by age 1 year and $50 \%$ by age 2 years; without access to life-saving drugs; including antiretroviral [1]. As of 2018, 1.7 million children are estimated to be living with the HIV disease globally; $91 \%$ of them are in the sub-Saharan Africa region. Also, the region accounted for $88.8 \%(146,000)$ of the total global new infection, and $87 \%$ of the global AIDS-related death; among children $0-14$ years [2].

Globally, new infection in children has been attributed largely to mother to child transmission. Prevention of Mother to Child Transmission (PMTCT) programs provides a range of services to women and infants to prevent HIV infection, reduce perinatal, intrapartum, and postnatal HIV transmission and curb AIDS-related death. A critical part of this is the Early Infant Diagnosis (EID) of HIV. Early Infant Diagnosis of HIV is very critical as it aids the prompt identification of infants infected with the virus for the timely commencement of the lifesaving antiretroviral (ART) drugs. This is further emphasized with the evidence of a $75 \%$ reduction in disease progression and a $76 \%$ reduction in HIV mortality in infants attributed to early diagnosis of HIV and early commencement on ART [3].

EID involves the detection of HIV infection in children less than 18 months using the nucleic acid based virological assay. This is specific, reliable, and recommended by the World Health Organization (WHO). Traditionally, the virological assay is provided by centralized PCR laboratories, however, bedeviled by several challenges. With only $58.7 \%$ of HIV Exposed Infants having access to virological test within the first 2 months of life globally in 2018, [2] the challenges with access to EID is obvious. These challenges have been documented in many 
studies to include loss of samples, long turnaround time (TAT), backlogs in sample processing, inefficient sample transport, long equipment downtime, and stock out of reagents among others [4]. This continues to contribute to high loss to follow-up (LTFU) and consequently infected infants are not identified and do not receive the treatment they need. Even though these challenges are similar, however, the impacts are to varying degrees across the different regions and countries.

In 2018, Nigeria has the highest new infection and AIDS-related death in children (0-14 years), globally. However, only $23.8 \%$ of HIV-exposed infants received an EID test and $35 \%$ of children living with HIV received treatment (UNAIDS, 2019). Virological testing for Early Infant Diagnosis is provided through centralized PCR laboratories and relying on the sample referral network for the transportation of samples and return of results. The earliest return of results from the centralized laboratory has been documented as 3 months and 5.6 months, even with $81.7 \%$ and $62.3 \%$ collection of DBS samples within the stipulated 2 months respectively [5,6]. Documented challenges with EID are similar to other sub-Saharan countries [7].

Point of care (POC) technologies provide a platform for the provision of virological based testing outside the traditional centralized laboratories hence, a means to improve access to Early Infant Diagnosis (EID). This has been recommended as a key game-changer in achieving a prompt diagnosis of HIV in infants and timely initiation on treatment [8]. Point of Care tools are tests which can be carried out near the patient or at the treatment facility, thereby allowing for prompt diagnosis and/or client management [9]. It is usually rapid in providing test results with resultant positive impact on diagnosis, patient management and disease surveillance. In resource-limited settings, studies have identified the restricted availability of laboratory -based clinical management as part of major factors causing the lack of qualified health care and poor disease outcomes [10]. To bridge this gap in many disease epidemic, POC test has been employed to mitigate unmet diagnostic capabilities. The use of the POC test for high-burden infectious disease in endemic region has been estimated to save over one million lives annually due to improved clinical management [11].

For Early Infant Diagnosis of HIV infection, several benefits have been alluded to the POC technologies. These include-shorter test Turnaround Time (TAT) which allow results to be returned during the same visit, thereby significantly reducing Loss to Follow Up (LFTU) and allows for immediate clinical management decisions to impact patient care and improve patient retention; reduction in the burden of managing an extensive sample transportation network, particularly in hard-toreach places; and ease of use as it can be operated by health workers lacking specialized laboratory skills. In Mozambique, the introduction of the point of care (POC) EID testing was found to have a significant impact on TAT-0 days TAT with POC compared with 127 days with the standard of care (POC) using the centralized laboratories. Result return was $95 \%$ and $65 \%$ with the POC and the SOC, respectively. While $89.7 \%$ of children were initiated in the POC EID arm, an abysmal $12.8 \%$ were initiated on the SOC arm [12]. Another retrospective study across eight (8) African countries indicates, $92.3 \%$ prompt ART initiation in HIV positive infants in the POC arm and $43.3 \%$ in the SOC arm. Result return to guardians/parent of infants was $98.3 \%$ and $18.7 \%$ in the POC and SOC arms respectively [13].

Despite the gaps in Early Infant Diagnosis of HIV infections and the high AIDS related death rate among infants and children, Nigeria is yet to introduce the Point of Care EID; notwithstanding its attendant benefit to address these challenges as seen in other sub-Saharan Africa countries. Also, no study has been conducted on the view of healthcare workers on the introduction and acceptability of a POC for EID in Nigeria. With the aim of assessing the standard of care and the acceptability of POC for the provision of EID in Nigeria, a descriptive cross-sectional survey was conducted across selected healthcare facilities. The opinion of healthcare workers was sought on the need and possible introduction of Point of Care (POC) Early Infant Diagnosis (EID) in Nigeria. It is hoped that this insight will provide perspectives on the current situation and challenges with the standard of care; the need for the Point of Care EID, its acceptability to healthcare worker 
hence; necessary information to guide its introduction and implementation in Nigeria.

\section{Materials and Methods}

\section{Study Setting}

The study was conducted across eight (8) healthcare facilities in three (3) states-Kaduna, Kogi, and Benue-located within the north-west and north-central geopolitical zone of Nigeria states. The healthcare facilities provide Early Infant Diagnosis through sample referral to the Centralized PCR laboratories. Also, the location of the healthcare facilities cuts across urban, peri-urban, and rural areas; as well as the three (3) levels of the health systems in NigeriaPrimary, Secondary, and Tertiary healthcare levels.

\section{Study Population}

The study population were healthcare workers with direct or indirect responsibilities linking to the provision or use of EID. All healthcare workers were located within the north-east and north-west region of Nigeria.

\section{Sampling}

Health facilities were selected across the 3 states based on 1-year historical volume of HIV Exposed Infants accessing virological testing. Purposive sampling method was employed in recruiting participants. Only health care workers with direct or indirect responsibilities linking with the provision or use of EID within the selected health facilities were recruited for the study.

\section{Study Design}

This was a descriptive cross-sectional survey of the opinion of frontline healthcare workers across 8 healthcare facilities in the north-west and north-central Nigeria; on Early Infant Diagnosis (EID). Healthcare worker's opinion was abstracted through the administration of a structured questionnaire. Healthcare facilities were selected to cut across the urban, peri-urban, and rural areas as well as the 3 levels of healthcare systems in Nigeria - Primary, Secondary, and Tertiary.

\section{Study Instruments}

The study instrument was a structured selfadministered questionnaire which was used to abstract healthcare workers opinion to provide data as it relates to the Standard of Care for EID, current challenges as well as need and acceptability of a Point of Care for EID. Information including designation, location of practice, gender, and qualification was documented. Informed Consent was secured before the administration of the questionnaire and confidentiality ensured.

\section{Data Management and Analysis}

Qualitative data were extracted from the completed structured self-administered questionnaires. Descriptive analysis was carried out to summarize the outcome measures and the background characteristics of all study participants using percentages for categorical variables and mean, median and standard deviations for quantitative variables. The results were presented as frequency tables, graphs, and percentages as appropriate. The analysis was both descriptive and inferential with values set at 95\% confidence level, a p-value of 0.05 was considered significant. All Analyses will be carried out using Stata version 14 (StataCorp, College Station, Texas, USA); and statistical level of significance set at $\mathrm{P}$-value $\leq 0.05$.

\section{Ethical Considerations}

Ethical approvals were obtained from the Kaduna, Benue, and Kogi States Ministry of Health Research and Ethics Review Committee. Written informed consent was obtained from all participants before the administration of the questionnaire.

\section{Results}

\section{Socio-demographic Characteristics of Participants}

This study was conducted among 72 healthcare workers across 8 healthcare facilities in the 3 states of Kaduna, Kogi and Benue; located within north-west and north-east Nigeria. The questionnaire was selfadministered and only 61 participants completed and returned the questionnaire; giving a recovery rate of $84.7 \%$. Analysis of the reported designation by participants indicate the highest as Laboratory personnel and Nurses; 40.98\% and $26.3 \%$ respectively (See Table 2). As regards gender, $56 \%$ of the respondents were male and $44 \%$ were female (See Table 1). The years of experience of respondents (healthcare workers) ranges between 3-35 years; however, 
most respondents $(82.0 \%)$ have professional experience within 3-10 years (See Table 3). The educational qualification of respondents is largely tertiary level (98.3\%).

Table 1. Distribution of Respondents (Health Care Providers) Based on Gender

\begin{tabular}{|l|l|l|}
\hline Sex & Frequency $(\mathbf{n})$ & Percentage (\%) \\
\hline Male & 27 & 44.26 \\
\hline Female & 34 & 55.74 \\
\hline Total & 61 & 100.00 \\
\hline
\end{tabular}

$\bar{x}=30.5, \tilde{x}=30.5$

Table 2. Distribution of Respondents (Health Care Providers) Based on Designation

\begin{tabular}{|l|l|c|}
\hline Designation & Frequency (n) & Percentage (\%) \\
\hline Doctor & 1 & 0.02 \\
\hline Nurse & 16 & 26.23 \\
\hline Pharmacist & 6 & 9.84 \\
\hline Laboratory Scientist & 25 & 40.98 \\
\hline Community Health Extension Workers (CHEW) & 4 & 6.56 \\
\hline Others & 9 & 14.75 \\
\hline Total & $\mathbf{6 1}$ & $\mathbf{1 0 0 . 0 0}$ \\
\hline
\end{tabular}

Table 3. Distribution of Respondents (Health Care Providers) Based on Gender

\begin{tabular}{|l|l|l|}
\hline Year(s) of Experience & Frequency $(\mathbf{n})$ & Percentage (\%) \\
\hline $1-5$ & 37 & 60.66 \\
\hline $6-10$ & 13 & 21.31 \\
\hline $11-15$ & 3 & 4.92 \\
\hline $16-20$ & 3 & 4.92 \\
\hline $21-25$ & 1 & 1.64 \\
\hline $26-30$ & 2 & 3.28 \\
\hline$>30$ & 2 & 3.28 \\
\hline Total & $\mathbf{6 1}$ & $\mathbf{1 0 0 . 0 0}$ \\
\hline
\end{tabular}

$$
\bar{x}=30.5, \tilde{x}=30.5
$$

Table 4. Health Care Providers Opinion on Current EID Processes and the Need for POC EID

\begin{tabular}{|l|l|l|l|}
\hline Questions & Options & Responses (n) & Percentage (\%) \\
\hline \multirow{2}{*}{$\begin{array}{l}\text { Does your job involve working on the Early } \\
\text { Infant Diagnosis of HIV exposed Infant }\end{array}$} & Yes & 43 & 79.6 \\
\cline { 2 - 4 } & No & 11 & 20.4 \\
\cline { 2 - 4 } & No Response & 7 & 11.5 \\
\hline \multirow{2}{*}{$\begin{array}{l}\text { In your opinion, is there a need for Early } \\
\text { Infant Diagnosis? }\end{array}$} & Yes & 57 & 93.4 \\
\cline { 2 - 4 } & No & 0 & 0.0 \\
\cline { 2 - 4 } & No Response & 4 & 6.6 \\
\hline \multirow{2}{*}{$\begin{array}{l}\text { Is the PCR test for Early Infant Diagnosis } \\
\text { carried out in your facility? }\end{array}$} & Yes & 0 & 0.0 \\
\cline { 2 - 4 } & No & 52 & 85.3 \\
\cline { 2 - 4 } & No Response & 9 & 14.7 \\
\hline \multirow{2}{*}{$\begin{array}{l}\text { How often do you send Dried Blood } \\
\text { laboratory? }\end{array}$} & Weekly & 31 & 50.8 \\
\cline { 2 - 4 } & Every 2 weeks & 18 & 29.5 \\
\cline { 2 - 4 } & $>2$ weeks & 4 & 6.6 \\
\cline { 2 - 4 } & No Response & 8 & 13.1 \\
\hline & $1-2$ weeks & 6 & 9.8 \\
\hline
\end{tabular}




\begin{tabular}{|c|c|c|c|}
\hline \multirow{4}{*}{$\begin{array}{l}\text { How long does it typically take to get results } \\
\text { for EID? }\end{array}$} & $3-4$ weeks & 19 & 31.2 \\
\hline & $5-6$ weeks & 10 & 16.4 \\
\hline & $>6$ weeks & 18 & 29.5 \\
\hline & No Response & 8 & 13.1 \\
\hline \multirow{5}{*}{$\begin{array}{l}\text { What is the average distance (in Kilometers) } \\
\text { from your facility to the PCR laboratory? }\end{array}$} & $<100 \mathrm{~km}$ & 14 & 22.9 \\
\hline & $101-102 \mathrm{~km}$ & 12 & 19.7 \\
\hline & $201-400 \mathrm{~km}$ & 7 & 11.5 \\
\hline & $>400 \mathrm{~km}$ & 19 & 31.1 \\
\hline & No Response & 9 & 14.8 \\
\hline \multirow{5}{*}{$\begin{array}{l}\text { What is the average number of Dried Blood } \\
\text { Specimen you collect within a week? }\end{array}$} & $<10$ & 39 & 63.9 \\
\hline & $10-49$ & 10 & 16.4 \\
\hline & $50-100$ & 4 & 6.6 \\
\hline & $>100$ & 0 & 0.0 \\
\hline & No Response & 8 & 13.1 \\
\hline \multirow{5}{*}{$\begin{array}{l}\text { What issue have you identified with the } \\
\text { conduct of EID in your facility }\end{array}$} & Stock out & 9 & 14.8 \\
\hline & Long TAT & 16 & 26.2 \\
\hline & Distance to the PCR Lab & 21 & 34.4 \\
\hline & Others & 0 & 0.0 \\
\hline & No Response & 15 & 24.6 \\
\hline \multirow{3}{*}{$\begin{array}{l}\text { Are you aware of a Point of Care (POC) test } \\
\text { for Early Infant Diagnosis? }\end{array}$} & Yes & 42 & 68.9 \\
\hline & No & 12 & 19.7 \\
\hline & No Response & 7 & 11.4 \\
\hline \multirow{4}{*}{$\begin{array}{l}\text { Would the introduction of a Point of Care } \\
\text { (POC) Early Infant Diagnosis (EID) be } \\
\text { beneficial? }\end{array}$} & Yes & 46 & 75.4 \\
\hline & No & 0 & 0.0 \\
\hline & Not Sure & 5 & 8.2 \\
\hline & No Response & 10 & 16.4 \\
\hline \multirow{6}{*}{$\begin{array}{l}\text { On a scale of } 1-5,(5 \text { being the highest and } \\
1 \text { the lowest), how would you rate the need } \\
\text { for a Point of Care (POC) equipment on } \\
\text { Early Infant Diagnosis (EID) in your facility }\end{array}$} & 5 & 15 & 24.6 \\
\hline & 4 & 13 & 21.3 \\
\hline & 3 & 13 & 21.3 \\
\hline & 2 & 7 & 11.5 \\
\hline & 1 & 2 & 3.3 \\
\hline & No Response & 11 & 18.0 \\
\hline \multirow{4}{*}{$\begin{array}{l}\text { Would the use of the Point of Care increase } \\
\text { patient uptake of EID? }\end{array}$} & Yes & 44 & 72.1 \\
\hline & No & 2 & 3.3 \\
\hline & Not Sure & 8 & 13.1 \\
\hline & No Response & 7 & 11.5 \\
\hline \multirow{4}{*}{$\begin{array}{l}\text { Which would be your preferred method for } \\
\text { EID testing within your facility? }\end{array}$} & Point of Care & 7 & 75.4 \\
\hline & PCR Laboratory & 21 & 0.0 \\
\hline & Both & 21 & 8.2 \\
\hline & No Response & 12 & 16.4 \\
\hline
\end{tabular}




\section{Opinion on the Current Processes for the Standard of Care}

Analysis of participants' responses indicate that $100 \%$ of the respondents believes there is a need for Early Infant Diagnosis. Across the eight healthcare facilities; $87.3 \%$ submitted that the method for EID sample collection is Dried Blood Spot. (DBS) while $12.7 \%$ reported venous blood sample. $100 \%$ of respondents reported that the PCR laboratory is located outside the health facility hence they rely on sample transfer to the centralized PCR laboratory. On the frequency for sending samples to the PCR laboratory, $58.5 \%$ of respondents submitted that samples are sent weekly to the PCR laboratories, $34.0 \%$ biweekly and $7.5 \%$ greater than 2 weeks. The average turnaround time (TAT) for the receipt of EID results was reported as 3-4 weeks by $35.8 \%$ of respondent, $>6$ weeks by $34.0 \%$ of respondents, 5-6 weeks by $18.9 \%$ of respondents and 1-2 weeks by $11.3 \%$ of respondents. About $73.6 \%$ of the respondent reported an average of 10 DBS samples collected weekly. Greater number of respondent $(45.7 \%)$ identified distance to the PCR laboratories as a key issue affecting the conduct of EID. Other's issues identified by respondent include long TAT (34.8\%) and reagent stock out $(19.6 \%)$.

\section{Awareness and Need for Point of Care}

Based on the analysis of the responses; $77.8 \%$ of respondents are aware of the existence of a point of care platform for Early Infant Diagnosis; $23.2 \%$ of respondents are however not aware. In response to the question on the benefit of POC for EID; $90.2 \%$ of respondent believe it is beneficial, while $9.8 \%$ are not sure of the benefit of the POC for EID. No respondent has the opinion that the POC for EID is not beneficial. When asked to rate the need for a POC for EID on a scale of $1-5$, altogether $82 \%$ rated the need for POC for EID as $5(30 \%)$, $4(26 \%)$ and $3(26 \%)$. Opinion on the effect of POC use on EID uptake indicate that $81.5 \%$ of respondent believe that with the introduction and use of POC for EID there will be an increase in EID uptake.

\section{Discussion}

With the aim of assessing the current situation and attendant challenges with the standard of care for the provision of Early Infant Diagnosis

\section{DOI: 10.21522/TIJPH.2013.09.01.Art009}

as well as the need and acceptability of Point of Care testing; a descriptive cross-sectional survey was conducted across eight healthcare facilities. The survey was conducted among 72 healthcare workers using self-administered questionnaire with $84.7 \%$ (61) recovery rate.

Based on the respondents, the method for sample collection across the healthcare facility is mainly through the use of Dried Blood Spot (DBS). The use of the DBS is an innovation which is targeted at mitigating the challenges of strict sample transfer procedure required for venous blood. It is in line with the WHO recommendation and it is expected that with these challenges addressed there should be a significant reduction in the turnaround time. However, most respondents reported an average turnaround time of 3-4 weeks (34.0\%) and >6 weeks $(34.0 \%)$. In the Healthy Baby Initiative "Baby Shower "study conducted in south eastern Nigeria, [14] reported an average turnaround time of 5.6 months. In the same vein, [6] reported 3 months' average turnaround time in the MoMENt study conducted in north central Nigeria. This aligns with the opinion of the respondents and establishes the issue of long TAT with the standard of care in Nigeria.

According to the submission of the respondents, the major key issues identified were distance to the PCR laboratory (45.7\%) and long TAT (34.8\%). These aligns with the reported issues at the Moment study. One can easily relate to the challenge of distance to the centralized PCR laboratories in Nigeria. With a land mass of 923, $768 \mathrm{~km}$, several difficult terrains and long distances to the centralized PCR laboratories; accessibility is always a challenge. Out of the 52 participants that responded to the question on the average distance from the heath facility to the centralized PCR laboratories; $36.5 \%$ reported an average of $>400 \mathrm{~km}$, while $36.6 \%$ reported an average of $100-400 \mathrm{~km}$.

Despite the non-existence of the Point of Care platform for EID in Nigeria; it is of interest to note that $77.8 \%$ of respondents reported that they are aware of the existence of a POC for EID. On the possible benefit of POC for EID, 90.2\% of respondent reported that it would be beneficial, with $81.5 \%$ of respondents indicating that the introduction of the POC will increase the uptake of EID. With the attendant challenges of the SOC, alternatives to ensure the provision of 
timely EID and prompt commencement of treatment is a key concern of frontline healthcare workers. The analyzed responses above indicate the willingness and acceptability of the Point of care for Early Infant Diagnosis by healthcare workers.

One of the eluded benefits of the SOC is the ability to pool samples and analyze many samples at a time however, the POC can only analyze a minimal number of samples at a time. It therefore pertinent to note that the reported $<10$ Dried Blood Spot (DBS) collected per week by $73.5 \%$ participants who responded to the question affirm the applicability of the POC equipment in a lot of places.

\section{Conclusion}

With a focus on the opinion of frontline health care workers on the current situation and need/acceptability of Point of Care testing for the provision of Early Infant Diagnosis; this study concludes that there are attendant challenges with the SOC in Nigeria which has limited the uptake of EID, early diagnosis of HIV exposed infants and prompt commencement of antiretroviral therapy in infants. The Point of Care testing for EID is a viable and acceptable alternative to increase EID uptake and hence prompt commencement on treatment and saving of lives.

\section{References}

[1] WORLD HEALTH ORGANIZATION (WHO). Global Programme on AIDS, 1988. WHO Report Breastfeeding, breast milk and human immunodeficiency virus (HIV). Statement from the Consultation held in Geneva, 23-25 June, 1987. AIDS Action, (5), 1-2. Date of Access: 05/05/2020. https://pubmed.ncbi.nlm.nih.gov/12281628/.

[2] UNAIDS Data 2019. Date of Access: 06/05/2020 https://www.unaids.org/sites/default/files/media_ass et/2019-UNAIDS-data_en.pdf.

[3] WORLD HEALTH ORGASNIZATION, 2011. The interagency task team on the prevention and treatment of HIV infection in pregnant women, mothers and children (IATT) Annual meeting report (2-3 May 2011), Geneva, Switzerland). Date of Access: 01/03/2020.

https://apps.who.int/iris/bitstream/handle/10665/446 37/9789241501927_eng.pdf;jsessionid=92F8E333F D82CE0F5BE7461D59C7F1C7? sequence=1.

[4] Anisa Ghadrshenas, Yanis B. Amor, Joy Chang, Helen Dale. et. al. 2013. Improved access to early infant diagnosis is a critical part of a child-centric prevention of mother-to-child transmission agenda. AIDS 2013, 27 (Suppl. 2): S197-S205. Date of Access: 13/03/2020

https://journals.lww.com/aidsonline/Fulltext/2013/1 1002/Improved_access_to_early_infant_diagnosis_i s_a.8.aspx.

[5] Carlucci, J. G., Liu, Y., Friedman, H., Pelayo, B. E., Robelin, K., Sheldon, E. K., Clouse, K., \& Vermund, S. H. 2018. Attrition of HIV-exposed infants from early infant diagnosis services in lowand middle-income countries: a systematic review and meta-analysis. Journal of the International AIDS Society, 21(11), e25209. Date of Access: 15/5/2020 https://doi.org/10.1002/jia2.25209.

[6] Udochisom C. Anaba, Nadia A. Sam-Agudu, Habib O. Ramadhani, Nguavese Torbunde, Alash'le Abimiku, Patrick Dakum, Sani H. Aliyu, Manhattan Charurat, 2019. Missed opportunities for early infant diagnosis of HIV in rural North-Central Nigeria: A cascade analysis from the INSPIRE MoMent study. PLoS ONE. Date of Access: 15/5/2020. https://doi.org/10.1371/journal.pone.0220616.

[7] Isah HO, Ogum E, Cornelius LJ, Okundaye JN, Galadanci H, Charurat ME, et al. 2014. The MoMent Study: Client- and Community-Level Barriers to PMTCT Access and Uptake in Rural North-Central Nigeria. 8th INTEREST (International Workshop on HIV Treatment, Pathogenesis, and Prevention Research in Resource-Poor Settings) Workshop; Lusaka, Zambia. p. 45. Date of Access: 15/5/2020. http://regist2.virology-

education.com/abstractbook/2014_3.pdf.

[8] WORLD HEALTH ORGANIZATION (WHO) The treatment 2.0 framework for action: catalysing the next phase of treatment, care and support. (2011). The World Health Organization (WHO) publication 2011. Date of Access: 03/05/2020. https://apps.who.int/iris/bitstream/handle/10665/446 40/9789241501934_eng.pdf;jsessionid=01FAF23E0 5BA02AA84E195D24277E22D?sequence $=1$.

[9] Schito M, Peter TF, Cavanaugh S, Piatek AS, Young GJ, Alexander H, Coggin W, Domingo GJ, Ellenberger D, Ermantraut E, Jani IV, Katamba A, Palamountain KM, Essajee S, Dowdy DW. 2012. Opportunities and challenges for cost-efficient implementation of new point-of-care diagnostics for HIV and tuberculosis. J Infect Dis. 2012 May 15; 205 Suppl 2(): S169-80. Date of Access: 29/06/2020. https://academic.oup.com/jid/article/205/suppl_2/S1 69/805866.

[10] Urdea M, Penny LA, Olmsted SS, Giovanni MY, Kaspar P, Shepherd A, Wilson P, Dahl CA, 
Buchsbaum S, Moeller G, Hay Burgess DC, 2006. Requirements for high impact diagnostics in the developing world. Nature. 2006 Nov 23; 444 Suppl 1():73-9. Date of Access: 29/06/2020. https://www.nature.com/articles/nature05448.

[11] Burgess DCH, Wasserman J, Dahl CA, Girosi F, Olmsted SS, Keeler EB, et al. 2007. Estimating the Global Health Impact of Improved Diagnostic Tools for the Developing World. Santa Monica, CA: RAND Corporation; 2007. Date of Access: 29/06/2020. http://www.rand.org/pubs/research_briefs/RB9293.h tml.

[12] Jani IV, Meggi B, Loquiha O, et al. Effect of point-of-care early infant diagnosis on antiretroviral therapy initiation and retention of patients. AIDS. 2018;32(11):1453-1463. doi:10.1097/QAD.0000000000001846.

[13]Flavia Bianchi, Jennifer Cohn, Emma Sacks, Rebecca Bailey, Jean-Francois Lemaire and Rhoderick Machekano. 2019. Evaluation of a routine point-of-care intervention for early infant diagnosis of HIV: an observational study in eight African countries. The Lancet HIV Volume 6, Issue 6, June 2019, Pages e373-e38. Date Accessed: 15/5/2020. https://doi.org/10.1016/S2352-3018(19)30033-5.

[14]Pharr, Jennifer R., Obiefune, Michael C.; Ezeanolue, Chinenye O.; Osuji, Alice; Ogidi, Amaka G.; Gbadamosi, Semiu, Patel, Dina, Iwelunmor, Juliet; Yang, Wei; Ogedegbe, Gbenga; Ehiri, John E.; Sam-Agudu, Nadia A.; Ezeanolue, Echezona E. 2016. Linkage to Care, Early Infant Diagnosis, and Perinatal Transmission Among Infants Born to HIVInfected Nigerian Mothers: Evidence from the Healthy Beginning Initiative, JAIDS Journal of Acquired Immune Deficiency Syndromes: August 1, 2016 - Volume 72 - Issue - p S154-S160. Date of Access:

5/04/2020.https://doi.org/10.1097/QAI.0000000000 001051. 